\title{
Acknowledgement of manuscript reviewers 2015
}

Nick Crofts ${ }^{1 *}$ and Euan Lawson ${ }^{2}$

\section{Contributing reviewers}

The editors of Harm Reduction Journal would like to thank all our reviewers who have contributed to the journal in Volume 12 (2015).

Robert Ali

Australia

Steve Allsop

Australia

Tasnim Azim

Bangladesh

Cas Barendregt

Netherlands

Brittany Barker

Canada

Andrew Barnes

USA

Angela Bazzi

USA

Lynne Belle-Isle

Canada

Ricky Bluthenthal

USA

Stephan Bongard

Germany

Susan Boyd

Canada

Jamie Brown

UK

\begin{abstract}
Joanne Bryant
\end{abstract}
Australia

Brent Caldwell

New Zealand

Tessa Cheng

Canada

Stephen Corson

UK

Constanza Daigre

Spain

Lucia D'ambruoso

UK

\section{Caitlin Davey}

Canada

Peter Davidson

USA

Colleen Dell

Canada

John Derricott

UK

Don Des Jarlais

US

Elinor Dickie

UK
Ernest Drucker

USA

Nadia Fairbairn

Canada

Michael Farrell

Australia

Gabriele Fischer

Austria

Niamh Fitzgerald

UK

Chris Ford

UK

Vivian Go

USA

Shira Goldenberg

USA

Lauretta Grau

USA

Mauro Guarinieri

Vietnam

Andrew Guise

UK

Wayne Hall

Australia

\footnotetext{
* Correspondence: nick.crofts@unimelb.edu.au

${ }^{1}$ The Melbourne School of Population and Global Health, Melbourne,

Australia

Full list of author information is available at the end of the article
} 
Theodore Hammett

USA

\section{Magdalena Harris}

UK

Kanna Hayashi

Evelyn Hearne

Ireland

Peter Higgs

Australia

Martin Iguchi

USA

Ehsan Jozaghi

Canada

Bengt Kayser

Switzerland

Ken Kirkwood

Canada

Irma Kirtadze

Georgia

Andrea Krüsi

Canada

M Suresh Kumar

India

Pritika Kumar

US

Margot Kuo

Canada

Jih-Heng Li

Taiwan

Tim Mackey

USA

Olivier Maguet

France

Jagadish Mahanta

India

Marcus Martens

Germany

Catriona Matheson

UK

Andrew Mcauley

UK

Peter Meylakhs

Russian Federation
Ingo Michels

Germany

Alison Munro

UK

Bronwyn Myers

South Africa

David A L Newcombe

New Zealand

Robert Newman

USA

Suzanne Nielsen

Australia

Daniel O'Keefe

Australia

David Otiashvili

Georgia

Stephen Pan

Canada

Dana Paquette

Canada

Vibhu Paudyal

UK

Einat Peles

Israel

Christiane Pflanz-Sinclair

UK

Carl Phillips

USA

Eileen Pitpitan

USA

Riccardo Polosa

Italy

Seyed Radfar Ramin

Iran

Afarin Rahimi-Movaghar Iran

Sanjeev Raj Neupane

Nepal

Teresita Rocha

Mexico

Elise Roy

Canada

Riza Sarasvita

Indonesia
Andrew Sceibe

South Africa

Sivasubramaniam Selvaraj

UK

Janie Sheridan

New Zealand

Andrew Smirnov

Australia

Richard Steen

Italy

Carol Strike

Canada

Victoria Sublette

Australia

Ave Talu

Estonia

Andy Tan

USA

Johannes Thrul

USA

Lianping Ti

Canada

Ambros Uchtenhagen

Switzerland

Lianne Urada

US

Edwin Van Teijlingen

UK

Jack Wallace

Australia

Nick Walsh

Australia

Brooke West

USA

Lucas Wiessing

Portugal

Alex Wodak

Australia

Heather Worth

Australia

Cheng-Fang Yen

Taiwan

Oleg Yussopov

Kazakhstan 